\title{
Measurement of spin asymmetries in the electron impact ionisation of alkali atoms
}

\author{
G Baum, M Moede, W Raith and W Schröder† \\ Fakultät für Physik, Universität Bielefeld, D-4800 Bielefeld, Federal Republic of Germany
}

Received 22 May 1984, in final form 17 September 1984

\begin{abstract}
Using a GaAsP polarised electron source and state-selected atomic beams of lithium, sodium and potassium we investigated the spin dependence of the total ionisation cross section in the energy range from threshold to ten times the threshold value. For $\mathrm{Li}$ and $\mathrm{Na}$ the measured spin asymmetries are very similar, having as a function of energy $E$ a maximum of about 0.5 at twice the threshold energy $I$ and a fall off towards zero at higher energies proportional to $\ln (I / E)$. The potassium data are considerably lower in the region from threshold up to five times threshold energy, showing an asymmetry of about 0.25 there. With lithium the near-threshold range was probed with a narrow electron energy distribution $(\Delta E=0.17 \mathrm{eV}$ ). It was found that the spin asymmetry stays well below unity at threshold $\left(A_{l}=0.45\right)$ and has little dependence on energy in this region.
\end{abstract}

\section{Introduction}

For several years spin effects in impact ionisation have been investigated experimentally by different groups using crossed beams of spin-polarised electrons and spin-polarised atoms. For light atoms the observed spin effects are due to electron exchange in the collision and are quite pronounced in the energy range from threshold to about ten times threshold energy. Results have been reported for hydrogen (Alguard et al 1977, Gay et al 1982), lithium (Baum et al 1981), sodium (Hils et al 1982) and potassium (Hils and Kleinpoppen 1978). The individual groups used quite different methods for polarising the electron and atomic beams.

We have extended our earlier studies on $\mathrm{Li}$ (Baum et al 1981) to the light alkali atoms $\mathrm{Li}, \mathrm{Na}$ and $\mathrm{K}$ using improved experimental techniques for polarising the beams. A better stability in intensity and polarisation of the electron and the atomic beam led to reduced systematic errors in the determination of the asymmetry. Thus our new results are more precise. All the results presented in this paper were obtained with the same apparatus and with the same experimental procedures.

The quantity which is determined in our investigations is the spin asymmetry $A_{I}$. It is obtained by measuring the beam polarisations $P_{\mathrm{e}}$ and $P_{\mathrm{a}}$, and by observing ion signal rates, $N_{\uparrow \downarrow}$ and $N_{\uparrow \uparrow}$, for antiparallel and parallel spin configurations of the two beams respectively.

$$
A_{I}=\frac{1}{P_{\mathrm{e}} P_{\mathrm{a}}} \frac{N_{\downarrow \uparrow}-N_{\uparrow \uparrow}}{N_{\downarrow \uparrow}+N_{\uparrow \uparrow}} .
$$

† Present address: LITEF (Litton Technische Werke), D-7800 Freiburg, Federal Republic of Germany. 
The asymmetry $A_{I}$ is related to the singlet $\left(\sigma_{s}\right)$ and triplet $\left(\sigma_{t}\right)$ ionisation cross sections by $\left.A_{I}=\left(\sigma_{\mathrm{s}}-\sigma_{\mathrm{t}}\right) / \sigma_{\mathrm{s}}+3 \sigma_{\mathrm{t}}\right)$. The total ionisation cross section is given by $\sigma=\frac{1}{4} \sigma_{\mathrm{s}}+\frac{3}{4} \sigma_{\mathrm{t}}$. Thus the asymmetry is a relative measure of singlet and triplet contributions. Pure singlet ionisation corresponds to $A_{I}=1$, pure triplet to $A_{I}=-\frac{1}{3}$.

\section{Experiment}

The main parts of the apparatus were already described in our previous paper (Baum et al 1981). Here the emphasis will lie on information about additions and changes that were made since then. A schematic diagram of the improved experimental set up is shown in figure 1.

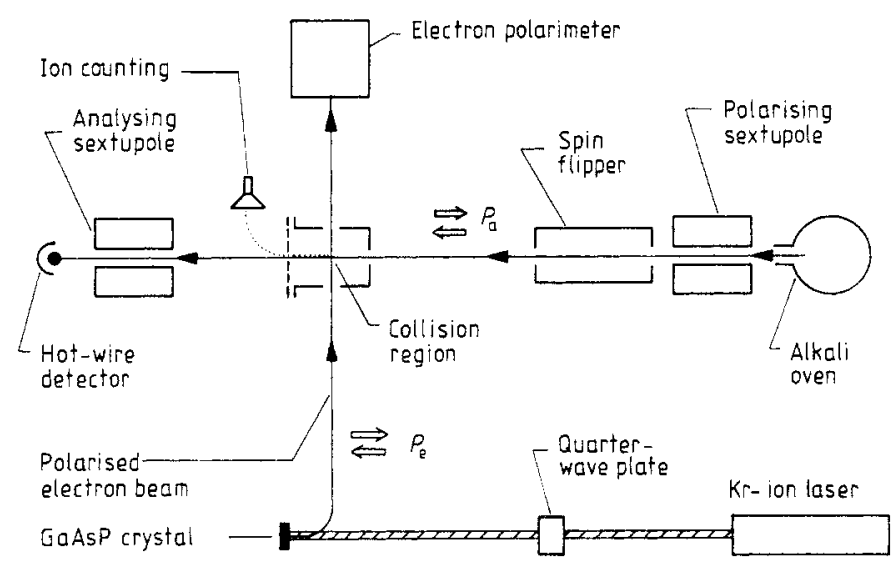

Figure 1. Schematic diagram of the experiment.

The polarised electron beam is now produced by photoemission from a GaAsP crystal using circularly polarised light. The (100) cut crystal is cleaned by a standard procedure (cf Conrath et al 1979) before its installation into the vacuum chamber. Once an ultra-high vacuum is obtained additional cleaning is achieved by heating, going through several temperature cycles, up to $650^{\circ} \mathrm{C}$. The crystal is activated by evaporating caesium from a dispenser (SAES Getters, Milano) onto the surface until a maximum in the photocurrent is observed. The application of oxygen results in an increase of the current to a slightly higher maximum. During operation of the source caesium is dispensed continuously at a reduced evaporation rate. This provides stability in intensity and polarisation of the electron beam over many hours. The light source used is a $\mathrm{Kr}$-ion laser operating on the $647 \mathrm{~nm}$ line. The light beam is focused onto the crystal surface to a spot of $0.2 \mathrm{~mm}$ diameter. A quarter-wave plate provides the circular polarisation. It can be rotated around the axis of the light beam by means of a stepping motor allowing an easy and computer-controllable reversal of the electron polarisation. An electrostatic spherical deflector bends the electron beam through $90^{\circ}$ (without introducing energy discrimination) and directs the electrons towards the collision region. Thereby the original longitudinal polarisation of the beam is changed to a transverse one. 
In general there is no critical procedure in the preparation of the source and it is no problem to obtain a stable polarised electron beam within a short time. Typical polarisation values, as measured by Mott scattering, are in the range 0.33 to 0.41 (depending on the crystal and its history), the energy spread is about $0.25 \mathrm{eV}$, and the quantum yield amounts to values around $10^{-3}$. The electron currents, adusted through the light intensity, ranged from $10 \mathrm{nA}$ to $2 \mu \mathrm{A}$ and were set according to the requirements of the investigation. The crystal is operated at room temperature. Overoxidation usually leads to a reduced energy width. Together with cooling by liquid nitrogen an energy width of $0.15 \mathrm{eV}$ is achieved. More details of the source are described elsewhere (Schröder 1982).

The atomic beam from a thermal effusion source is polarised by high-field state selection in a sextupole magnet. Beyond the magnet the polarised beam traverses the spin flipper, the collision chamber, and the analysing sextupole magnet before hitting the hot-wire detector which measures the transmitted beam intensity (figure 1). The polarising magnet consists of two segments, each of $7.6 \mathrm{~cm}$ length, $0.32 \mathrm{~cm}$ central bore diameter, and about $0.9 \mathrm{~T}$ pole-tip field. An identical magnet, located downstream of the collision region, serves as an analyser for the atomic beam polarisation. A perfect high-field state selector would lead to a low-field electronic polarisation of $P_{\mathrm{a}}=$ $1 /(2 I+1)$ (cf Hughes et al 1972), where $I$ is the nuclear spin of the alkali atom $(I=1$, $\frac{3}{2}$, and $\frac{3}{2}$ for the investigated atoms ${ }^{6} \mathrm{Li},{ }^{23} \mathrm{Na}$ and ${ }^{39} \mathrm{~K}$ respectively). Non-perfect selection resulting from insufficient separation of the $m_{\mathrm{s}}$ states in the given magnetic field will reduce the polarisation further, in particular for sodium with its strong coupling between nuclear and electronic spin. Therefore, a direct measurement of $P_{\mathrm{a}}$ is essential.

The spin flipper (Schröder and Baum 1983) is used for reversal of the atomic beam polarisation in the course of the scattering experiment and for making a measurement of this polarisation possible. To accomplish polarisation reversal, a small $(0.1 \mathrm{mT})$ transverse magnetic field is applied under computer control at the centre of the spin flipper, causing atoms to go from a diabatic passage to an adiabatic passage through the device. The applied magnetic field has no effect on other beam parameters, but changes only the spin orientation, as was confirmed by measurements with unpolarised electrons. For determining the beam polarisation we compare atomic beam intensities behind the analysing magnet for the spin flipper operating in the adiabatic mode $\left(i^{\mathrm{A}}\right)$ and in the diabatic mode $\left(i^{D}\right)$. In good approximation there is an equal population within the group of hyperfine states with $m_{s}=\frac{1}{2}$ and within the group of states with $m_{\mathrm{s}}=-\frac{1}{2}$ for the polarisation method employed here. The atomic polarisation is then given by (cf equation (15) of the paper by Schröder and Baum 1983):

$$
P_{\mathrm{a}}=\left(1-i^{\mathrm{D}} / i^{\mathrm{A}}\right) /\left[(2 I+1) i^{\mathrm{D}} / i^{\mathrm{A}}-(2 I-1)\right] .
$$

For ${ }^{6} \mathrm{Li},{ }^{23} \mathrm{Na}$ and ${ }^{39} \mathrm{~K}$ we measure typical values of $P_{\mathrm{a}}=0.29,0.185$ and 0.22 , respectively. This is in accord with expectations based on trajectory calculations, as well as with estimates of effects from strongly coupled hyperfine states. The beam density in the collision region was typically $10^{9}$ atoms $/ \mathrm{cm}^{3}$ for these ionisation measurements. The atomic beam is quite stable in intensity and polarisation over many hours. Deposition of alkali metal in the entrance portion of the magnet bore gradually reduces the beam polarisation by a small amount $(0.015)$ which we explain by the restriction of atomic paths to the region where the magnetic field is weak and hyperfine coupling effects influence the state selection. The beam polarisation also depends slightly on 
the oven temperature because the associated change of beam velocity leads to a slight change in the state selection.

At each chosen energy data are accumulated in sequential sets, each consisting of six runs with the following pattern of relative polarisation orientations $(\uparrow \downarrow, \uparrow \uparrow$, $\uparrow-, \downarrow \uparrow, \downarrow \downarrow, \downarrow-)$. The arrows indicate in order the spin directions of electron and atomic beam and the hyphen symbolises the closed atomic beam flag for measurements of the background. (The beam flag is located between spin flipper and collision region.) Each run lasts for the same length of time, typically 1 to $2 \mathrm{~s}$, with the gate of the corresponding scaler open for data accumulation. Beam parameters were changed under computer control and $0.5 \mathrm{~s}$ were allowed to turn the quarter-wave plate, switch the current in the spin flipper, or operate the beam flag between runs. About a million counts are collected at each energy point. During data taking the electron polarisation is measured about every two to three hours, the atomic polarisation is recorded together with each data set; the energy calibration and energy width of the electron beam are checked as needed, quite frequently (at about $15 \mathrm{~min}$ intervals) during the threshold studies. In measuring the energy dependence of $A_{I}$, data at a reference point (near $10 \mathrm{eV}$ incident energy) are interspersed. For the reference point the absolute value of the asymmetry has been well determined. The counting rate asymmetry observed at the reference point then provides information on the product $P_{\mathrm{e}} P_{\mathrm{a}}$. The collinearity of the electron and atomic beam polarisations was checked by observing the asymmetry at the reference point while rotating the electron polarisation around the electron beam axis as well as rotating the atomic polarisation around a normal to the plane containing the beams. Both spin rotations covered a range of about $\pm 30^{\circ}$ and were accomplished by application of magnetic fields, for the electrons by a longitudinal field at the exit of the source chamber, and for the atoms by fields which change the direction of the magnetic guiding field in the collision region as wanted. (For each field setting the electron trajectories had to be optimised.) A systematic reduction of the raw asymmetry was observed at high counting rates $(\geqslant 10 \mathrm{kHz})$. This effect was attributed to onset of saturation in the spiral-shaped channel electron multiplier (Valvo B 318 BL-01) used for ion counting, in accordance with the manufactuer's specifications on gain versus counting rate. Therefore, data were taken with low counting rates $(\leqslant 3 \mathrm{kHz})$.

As shown in figure 3 the energy-dependent ionisation yield near threshold is extrapolated linearly to give the ionisation threshold value and a calibration of the energy scale. From the full set of data points on $Y(E)$, most of which lie outside of the frame of figure 3, we concluded that a linear extrapolation is justified. Using a power law of 1.127 , as suggested by Wannier's theory, would not lead to more reliable results since systematic errors due to changes in electron optics with energy cannot be ruled out. The error in scale calibration is estimated to be less than $0.05 \mathrm{eV}$. From the deviation of the $Y(E)$ curve from a linear behaviour close to threshold the energy width of the electron beam is extracted. The validity of this procedure was checked by independent $\Delta E$ measurements with a retarding potential method. The typical energy width of the electron beam is 0.25 to $0.3 \mathrm{eV}$. Contribution of background to the signal is negligible below $20 \mathrm{eV}$, except for the threshold region; above $20 \mathrm{eV}$ the background contribution resulting from ionisation of residual gas lies below $50 \%$. Because of slightly different electron currents associated with the electron polarisation directions, the raw asymmetries are calculated separately for each electron polarisation. They are found to be equal in their absolute values within 0.001 and are combined for the final results. Test measurements with unpolarised electrons indicated that instrumental asymmetries are smaller than 0.001 . 


\section{Results and discussion}

The results of our asymmetry measurements on the alkali-metal atoms lithium, sodium, and potassium are shown in figure 2. At each data point the statistical error is indicated. The calibration uncertainty in the vertical scale, resulting from the uncertainties connected with the measurement of the atomic beam and electron beam polarisation are given in the figure caption.

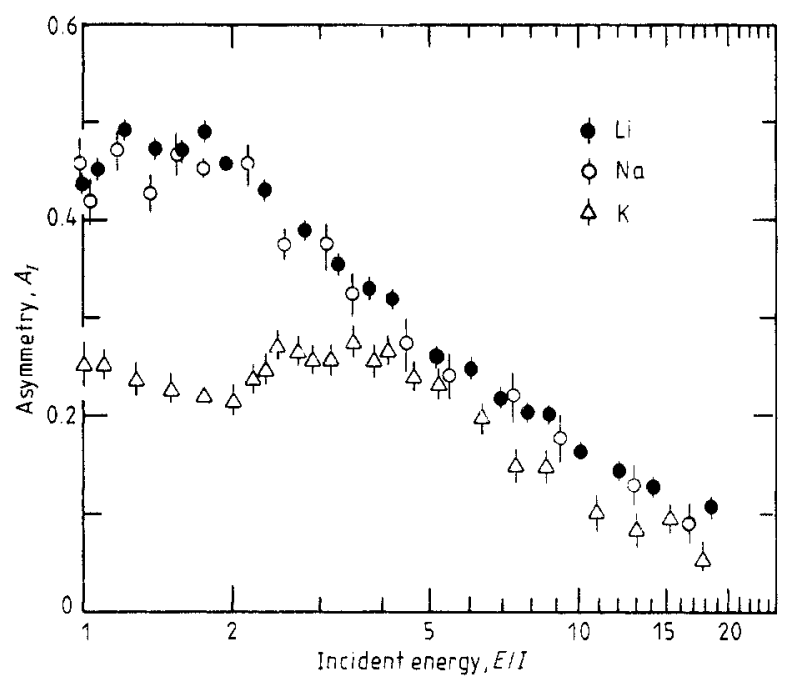

Figure 2. Measured values of the ionisation asymmetry $A_{l}$ as function of the incident energy $E$, in units of threshold energy $I$, for lithium $(I=5.39 \mathrm{eV})$, sodium $(I=5.14 \mathrm{eV})$, and potassium $(I=4.34 \mathrm{eV})$. The typical energy spread in the electron beams was $0.3 \mathrm{eV}$. Error bars show only the statistical uncertainty. In addition, we estimated a systematic error, common to the respective data points, which amounts to $\pm 6 \%$ for $\mathrm{Li}, \pm{ }_{10}^{5} \%$ for $\mathrm{Na}$, and $\pm 8 \%$ for $\mathrm{K}$.

For lithium the asymmetry peaks near $E / I=2$ with values now close to $A=0.5$. Towards threshold there is a slight decrease of the asymmetry; towards higher energies the asymmetry decreases nearly linearly in the representation of figure 2 in which a logarithmic energy scale is chosen. The ionisation asymmetry for sodium is nearly identical to the one of lithium, in its absolute values as well as in its energy dependence. The atomic core does not seem to be relevant for the size of the observed spin dependence in these cases. For potassium, however, there is a substantial deviation from this behaviour. In the energy range from threshold to $E / I=4$ the potassium asymmetrias are as much as a factor of a half smaller. At $E / I=5$ the potassium values merge with those of the two lighter alkalis. The difference at low energies might be due to the influence of spin-orbit coupling. As spin-orbit effects (e.g. the one revealed by the fine-structure splitting) depend quadratically on the nuclear charge $Z$, it is conceivable that their effects begin to show up prominently and abruptly in the asymmetry of potassium while absent in the lighter alkali atoms.

Our measurements can be compared with results of other groups on hydrogen, sodium and potassium. The hydrogen data (Alguard et al 1977, Gay et al 1982) closely follow our $\mathrm{Li}$ and $\mathrm{Na}$ asymmetry values, but are consistently lower with an average 
difference of 0.05 . The errors stated for $A_{I}$ of hydrogen, including statistical and systematic uncertainties, are twice as large as the ones for lithium shown in figure 2 . A direct comparison of results can be made for $\mathrm{Na}$ and $\mathrm{K}$. For $\mathrm{Na}$ a large discrepancy exists between our data and the results of Hils et al (1982) which are roughly a factor two smaller. Only about half of this discrepancy could be explained by the large systematic uncertainties in $P_{\mathrm{e}}$ and $P_{\mathrm{a}}$ stated by Hils et al. The overall energy dependence however is quite similar. The potassium data of Hils and Kleinpoppen (1978) have rather large error bars but within these they are in agreement with our measurements, being overall slightly lower by a difference of 0.05 to 0.1 . The authors state that their asymmetry scale could be in error because of incomplete knowledge of the atomic beam polarisation.

In the energy range from threshold to about ten times threshold energy there are no theoretical approaches which treat the ionisation process correctly. Basically the discussion which was given in our earlier paper for lithium (Baum et al 1981) also applies here. The binary encounter model treats the electron-electron collision correctly, and the large positive asymmetry arising from these collisions forms the basis for a physical understanding of the observed asymmetry. Difficulties and ambiguities arise when approximations for the bound nature of one of the electrons have to be made. In addition, not all collisions are of the binary encounter type (Ehrhardt et al 1982). There are also processes connected with momentum transfer to the ion (recoil peak). The first Born approximation, besides being not stringently applicable in this energy region, leads to problems in the calculation of the phase difference between the direct and the exchange ionisation amplitude. This is caused by the fact that there is no $a b$ initio way in which the effective nuclear charge seen by each of the two outgoing electrons can be calculated. The phase difference strongly effects the spin asymmetry; depending on the arbitrarily chosen value the predicted asymmetry can cover a range from zero to quite large positive values.

At the ionisation threshold the GaAsP photoemission source allowed measurements of the asymmetry with better energy resolution than possible with the field emission source (Baum et al 1981). For lithium the data points are shown in figure 3. The statistical error is indicated. The mean width of the electron energy distribution is $0.17 \mathrm{eV}$ (FWHM). Background contributions are present as is revealed by measurements below the ionisation threshold. The asymmetry of the background was measured and found to be consistent with zero. In the worst case the background fraction amounted to $40 \%$ of the observed signal. We obtained similar threshold results for potassium, but did not perform extensive threshold studies for sodium. Electron trajectory calculations indicate that in our apparatus the overlap of the electron beam with the atomic beam does not stay sufficiently constant in the energy regime of the threshold studies. Therefore we did not attempt to extract the energy exponent of the ionisation cross section for a comparison with power-law predictions.

As seen in figure 3 the asymmetry clearly shows no statistically significant dependence on energy near threshold $\dagger$. This implies that singlet and triplet processes have the same energy dependence. For the ratio of triplet to singlet cross sections at threshold our measurements give $r=0.25$. There is no fundamental reason why the asymmetry should approach unity at threshold, as had been concluded from a paper of Klar and Schlecht (1976). This point was already clarified in our earlier paper (Baum et al

$\uparrow$ No search was undertaken for an oscillatory behaviour of $A_{I}$ near threshold, as predicted by a Coulombdipole threshold theory (Temkin 1982) and experimentally tested by Kelley et al (1983). 


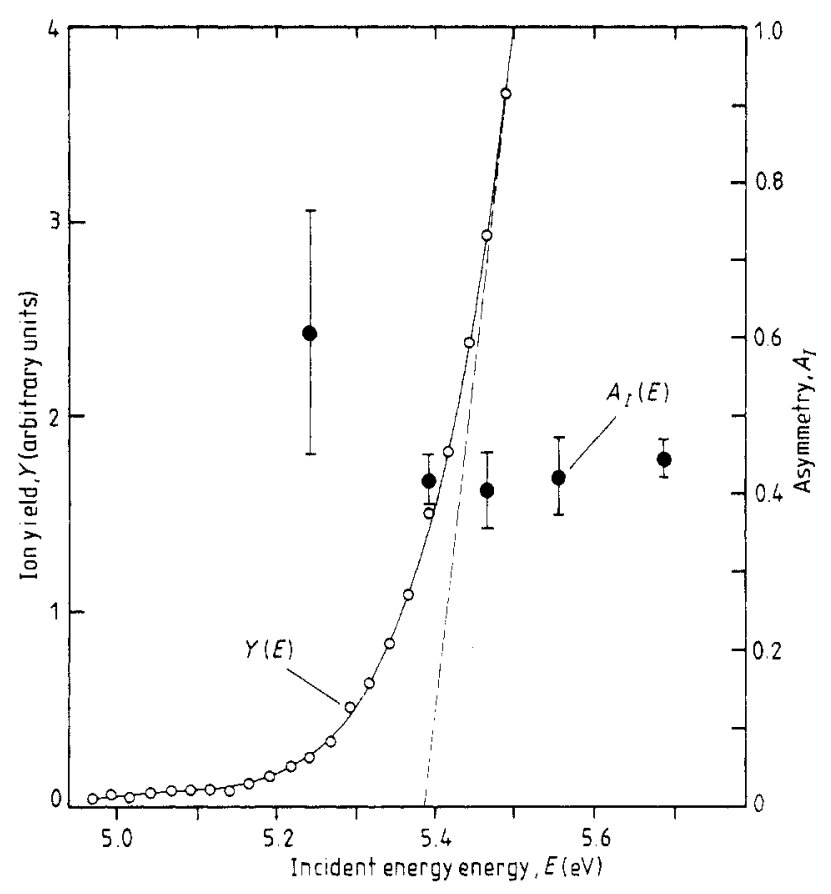

Figure 3. Measured ionisation asymmetry (full circles) and ion yield (open circles) as function of electron energy in the threshold region of lithium. The error bars give the statistical uncertainty. Only a small fraction of the data points on which the yield extrapolation to threshold energy (broken curve) is based fall into the figure frame. The mean energy width for the asymmetry data points is $0.17 \mathrm{eV}$ (FWHM).

1981). The dependence of the two-electron wavefunction on angular momentum, spin, and parity in the double escape at threshold was recently analysed in detail in the frame of the Wannier theory (Greene and Rau 1982, Stauffer 1982). The ratio $r$ gives information about the participating $\mathrm{L}$ waves, as singlet processes go with $\mathrm{S}$ waves (and higher even $\mathrm{L}$ waves) and triplet processes go with $\mathrm{P}$ waves (and higher odd $\mathrm{L}$ waves).

\section{Acknowledgments}

We thank Professor Dr E Reichert, Mainz, for kindly providing a GaAsP crystal which was used in part of these measurements. The work has been supported by the Ministry of Science and Research of Nordrhein-Westfalen and the Sonderforschungsbereich 'Polarisation and Correlation in Atomic Scattering Complexes' of the Deutsche Forschungsgemeinschaft.

\section{References}

Alguard M J, Hughes V W, Lubell M S and Wainwright P F 1977 Phys. Rev. Lett. 39 334-8

Baum G, Kisker E, Raith W, Schröder W, Sillmen U and Zenses D 1981 J. Phys. B: At. Mol. Phys. 14 4377-88

Conrath D, Heindorff T, Hermanni A, Ludwig N and Reichert E 1979 Appl. Phys. $20155-7$ 
Ehrhardt H, Fischer M, Jung K, Byron F W Jr, Joachain C J and Piraux B 1982 Phys. Rev. Lett. 48 1807-10 Gay T J, Fletcher G D, Alguard M J, Hughes V W, Wainwright P F and Lubell M S 1982 Phys. Rev. A 26 3664-7

Greene C H and Rau A R P 1982 Phys. Rev. Lett. 48 533-7

Hils D, Jitschin W and Kleinpoppen H 1982 J. Phys. B: At. Mol. Phys. 15 3347-57

Hils D and Kleinpoppen H 1978 J. Phys. B: At. Mol. Phys. 11 L283-7

Hughes V W, Long R L Jr, Lubell M S, Posner M and Raith W 1972 Phys. Rev. A 5 195-222

Kelley M H, Rogers W T, Celotta R J and Mielczarek S R 1983 Phys. Rev. Lett. 51 2191-3

Klar H and Schlecht W 1976 J. Phys. B: At. Mol. Phys. 9 1699-711

Schröder W 1982 Doctoral Thesis Universität Bielefeld (unpublished)

Schröder W and Baum G 1983 J. Phys. E: Sci. Instrum. 16 52-6

Stauffer A D 1982 Phys. Lett. 91A 114-6

Temkin A 1982 Phys. Rev. Lett. 49 365-8 